\title{
Un silence éloquent : narration et fantastique dans Fragoletta (1829) de Latouche et La Vénus d'Ille (1837) et Lokis (1869) de Mérimée
}

Nous proposons d'analyser trois œuvres, un roman de Latouche et deux contes de Mérimée, qui mettent en place une stratégie narrative similaire pour créer une tension entre le rationnel et l'irrationnel et produire un effet fantastique. Le parti pris narratif, qui sous-tend cette stratégie, est lié au statut paradoxal du narrateur dans le récit. Dans les trois textes, le lecteur se trouve face à un récit lacunaire, elliptique, à cause du refus (ou de l'impossibilité) du narrateur de livrer un sens ultime en interprétant les événements. Les non-dits et les blancs interprétatifs, ménagés dans le récit, qui oblitèrent le sens, produisent ainsi un va-et-vient entre le dicible et l'indicible. Il s'agit d'une sorte de "tactique du silence » ${ }^{1}$ qui participe d'une dynamique de l'énigme insoluble, du scandale intellectuel.

\section{Le silence et la rumeur}

Les trois œuvres en question s'attachent à décrire un objet fictionnel de nature fantastique : le corps hermaphrodite de Fragoletta, la statue animée de la Vénus et l'homme-bête dans Lokis. Cet objet devient le point focal dans lequel convergent plusieurs discours explicatifs qui restent inaboutis : la conceptualisation problématique de cet objet transgressif, inclassable et inintelligible, provoque une crise interprétative qui se traduit par une crise de la parole ${ }^{2}$. Cette parole inadéquate et défi-

Alexandra Jeleva - maître assistante au Département d'études romanes de l’Université de Sofia «St. Clément d'Ohrid ». Adresse pour correspondance : Université de Sofia «St. Clément d'Ohrid », boulevard Tsar Osvoboditel 15, bureau 160A, 1504, Sofia, Bulgarie ; e-mail ajel@abv.bg

1. Nous empruntons cette expression à Alain Corbin (voir : le chaptire intitulé « Les tactique du silence », in : Histoire du silence de la Renaissance à nos jours, Paris, Albin Michel, 2016, p. 127-144).

2. Nous ne citerons que quelques définitions du fantastique allant dans ce sens : " Le fantastique, c'est l'hésitation éprouvée par un être qui ne connaît que les lois naturelles, face à un événement en apparence surnaturel » (Tzvetan Todorov, Introduction à la littérature fantastique en France, Paris, Seuil, 
ciente débouche sur le silence, signalant par là l'échec de la rationalité. Max Picard avoue : "L'homme se tait de soi-même quand il voit un objet pour la première fois " (1954 : 55). Dans les trois textes, la représentation textuelle de cette crise explicative se présente comme une scission discursive qui oppose un discours rationaliste prétendant à l'efficacité absolue et un discours marqué de réticences et de non-dits qui manifeste, au contraire, une rationalité vacillante, consciente de ses limites. Dans son ouvrage Le monde du silence, Max Picard distingue d'une part la "parole » qui, procédant du logos, sait mettre des frontières conceptuelles et linguistiques et a partie liée avec le silence, et, d'autre part, la " rumeur » : une espèce de parole " amorphe " (1954 : 135) qui brouille les frontières et qui a besoin de se dérouler continuellement, de façon mécanique, pour remplir un vide (un silence) dont elle a peur et se prouver à elle-même qu'elle existe (Picard, $1954: 134-154$, passim). Nous avons emprunté à Max Picard la notion de "rumeur " pour désigner la parole vaine, le discours verbeux et sclérosé fait de clichés et de citations, qui s'efforce en vain de réduire l'inconnu au connu. À cette hypertrophie discursive s'oppose une rationalité plus modeste qui relève d'une parole qui ne cherche pas à dissimuler sa déficience explicative et qui relève - selon une expression que nous empruntons à Alain Corbin (2016 : 132) - d'un véritable art de la prudentia : ainsi que l'affirme La Rochefoucald, cité par Alain Corbin, «le silence est le parti le plus sûr de celui qui se défie de soi-même " (Corbin, 2016: 132). Dans les trois textes, l'interprétation rationaliste se solde par un échec. Dans Fragoletta, au discours épistémologiquement et esthétiquement conformiste que d'Hauteville tient devant la statue d'Hermaphrodite au musée de Naples, s'opposent, d'une part, l'argumentation subversive d'Éléonore, défendant la vraisemblance épistémologique et esthétique de l'hermaphrodite et, d'autre part, le silence révélateur de Camille qui confirme l'interprétation d'Éléonore - en apparence irrationnelle et invraisemblable - et signale la faillite du discours doxal. En voyant la statue et la réaction de d'Hauteville, elle s'enfonce dans le silence, parce qu'elle prend conscience de sa propre physiologie tératologique, cependant, plongés dans leur discussion, d'Hauteville et Éléonore ne cherchent pas à analyser son mutisme. La gnomorrhagie de M. de Peyrehorade et le bon sens épais du procureur du roi dans La Vénus d'Ille, ainsi que le matérialisme grossier du médecin dans Lokis, sont contrebalancés par la rationalité circonspecte du narrateur qui produit une parole parcimonieuse ("Je me gardai bien de critiquer son étymologie " (Mérimée, 1927 : $32)$; « Le matérialisme grossier du docteur me choquant au dernier point, je terminai brusquement l'entretien [...] » (Mérimée, 1993 : 250)). La parole d'Alphonse et du comte, réticente, entrecoupée de suspensions, est révélatrice d'une irrationalité

1970, p. 29) ; « [...] le Fantastique n'est pas, comme on le dit trop souvent, simple réaction compensatoire à l'impérialisme de la raison, mais bien un phénomène esthétique lié [...] à la crise de la raison » (Jean Fabre, Le miroir de sorcière, Paris, José Corti, 1992, p. 86) ; " Mais les textes fantastiques sont, eux, construits afin de montrer les diverses modalités d'une telle "mise en crise" du sens, pris entre l'avancée de la raison et l'irrationnel qui en cerne les territoires " (Roger Bozzetto, Territoires des fantastiques, Aixen-Provence, publication de l’Université de Provence, 1998, p. 5). 
sous-jacente. La réticence traduit l'embarras d'un locuteur, porteur d'une rationalité doxale, face à un phénomène inexplicable et en même temps, en validant la véracité et la bonne foi testimoniale de cette parole, consciente de son absurdité apparente, elle vraisemblabilise l'interprétation fantastique :

- Vous allez vous moquer de moi... Mais je ne sais ce que j’ai... je suis ensorcelé ! [...] Vous savez bien mon anneau ? poursuivit-il après un silence.

- Eh bien! On l'a pris?

- Non.

- En ce cas, vous l'avez?

- Non... je... je ne puis l'ôter du doigt de cette diable de Vénus.

- Bon! Vous n’avez pas tiré assez fort.

- Si fait... Mais la Vénus... elle a serré le doigt (Mérimée, 1927 : 36).

On relève la même parole contrainte, censurée par la rationalité, dans Lokis : «- J’ai voulu que vous fussiez témoin par vous-même... En votre qualité de savant, vous devez expliquer les énigmes... Pourquoi les animaux ont-ils peur de moi ? - En vérité, monsieur le comte, vous me faites l'honneur de me prendre pour un CEdipe » (Mérimée, $1993: 228$ ).

En se refusant à résoudre l'énigme, le narrateur révèle son statut paradoxal, qui se manifeste également dans Fragoletta et La Vénus d'Ille. La passivité interprétative du narrateur produit une narration inachevée, un sens éclaté. Fragoletta se termine sur le silence du moine qui est le seul témoin oculaire pouvant attester la nature anomale de Camille :

[...] quand il eut commencé d'écarter les vêtements qui couvraient la poitrine et les deux blessures encore sanglantes, il tressaillit. Il posa néanmoins à la hâte un premier appareil avant de s'éloigner et il séloigna les yeux baissés, le front rouge et le maintient trouble. Le secret de son trouble, la clé de la cellule où respirait encore la victime, le moine ne voulut jamais les confier quà son prieur et, le lendemain, au point du jour :

- Mes frères, dit le révérend prieur à deux laïques appelés pour ouvrir une tombe, il faut porter ce cadavre chez les sœurs de la Miséricorde (Latouche, 1867 : 344).

La Vénus d'Ille se termine sur le post-scriptum d'une lettre envoyée au narrateur par son ami M. de P. C'est dire que le narrateur finit par abandonner le récit, en laissant le mot de la fin à la parole d'autrui, à une instance narrative anonyme qui prend le relais pour conclure formellement le récit, sans élucider le mystère. Dans Lokis, le narrateur coupe net son récit ( - Et l'histoire est finie ? demanda Adélaïde. Finie! répondit le professeur d'une voix lugubre " (Mérimée, 1993 : 262)). De plus, le récit s'ouvre et se referme sur des points de conduite, comme pour marquer une rupture originelle du sens, une vacuité discursive et interprétative foncière qui débouche sur l'aporie et le silence, rendant patent l'échec de la parole rationnelle. Par 
son refus de livrer un récit achevé, l'instance narrative cautionne, par contrecoup, la lecture fantastique des événements.

\section{Les indices, les paralipses et le suspens}

L'inscription de l'objet fantastique dans le texte implique un parti pris scriptural qui oscille entre le dit et le non-dit. Cette écriture, génératrice d'une crise interprétative, se traduit plus concrètement par une dialectique manipulatrice des indices et des paralipses. Dans Figures III, G. Genette définit la paralipse comme « l'omission de telle action ou pensée importante du héros focal, que ni le héros, ni le narrateur ne peuvent ignorer, mais que le narrateur choisit de dissimuler au lecteur » (1972 : 212). C'est ainsi que le narrateur, dans Le meurtre de Roger Ackroyd, passe sous silence le fait qu'il est lui-même le meurtrier (1972 : 212). Distinguant soigneusement l'information donnée par un récit focalisé de l'interprétation que le lecteur lui donne, Gérard Genette emprunte à Roland Barthes la notion d'indices pour désigner « cet excès de l'information implicite sur l'information explicite » (1972 : 213) qui permet au lecteur de déchiffrer le sens malgré les paralipses.

Le jeu des indices et des paralipses, dans les trois textes qui font l'objet de cette analyse, relève d'une manipulation interprétative, car il ne s'agit pas seulement d'un déséquilibre entre l'information explicite et l'information implicite, compensé par le texte, mais d'une véritable dichotomisation explicative, suivant l'opposition conceptuelle rationnel/irrationnel. Le récit indiciaire est assumé par un narrateur rationnel, doxal, mais il infléchit la lecture dans le sens d'une interprétation irrationnelle qui affleure comme un arrière-plan signifiant jamais explicité, jamais assumé catégoriquement par ce même narrateur. Cette stratégie narrative ne permet que la reconstitution imparfaite d'un sens conjectural, du fait de la défection de l'instance narrative. Cette défection, découlant non pas d'une volonté, mais d'une incompétence interprétative du narrateur, produit un récit en suspens. Nous avons choisi ce mot pour désigner ce type de récit, car suspens signifie à la fois "indécision, incertitude » et "suspense ${ }^{3}$. En effet, l'économie du dit et du non-dit que nous avons jusqu'ici analysée comme génératrice d'une crise interprétative, présente également un deuxième aspect qui a trait au suspense et à la dynamique de l'énigme. Ainsi que l'observe Raphaël Baroni, la réticence apparaît comme un élément constitutif de la tension narrative :

Pour conclure ce rapide survol du traitement du suspense et de la curiosité par les théories de la réception, nous retiendrons certains points essentiels qui définiront à partir de maintenant le phénomène global de la tension narrative : la tension, sur le plan textuel, est le produit d'une réticence (discontinuité, retard, délai, dévoiement, etc.) qui induit

3. Cf. TLFi, article «Suspens ». TLFi : Trésor de la langue Française informatisé, http://www.atilf.fr, ATILF - CNRS\&Université de Lorraine. 
chez l'interprète une attente impatiente portant sur les informations qui tardent à être livrées ; cette impatience débouche sur une participation cognitive accrue, sous forme d'interrogations masquées et d'anticipations incertaines ; la réponse anticipée est infirmée ou confirmée lorsque survient enfin la réponse textuelle (2007 : 92).

Dans le récit fantastique, la réponse textuelle ne vient pas. On peut parler, dans ce cas, d'un suspense déceptif. Dans la préface de Fragoletta, l'auteur affirme d'emblée qu'il raconte " un mystère qu'il n'est pas tenu de comprendre » (Latouche, $1867: \mathrm{I})$; dans La Vénus d'Ille, à la fin de son récit, le narrateur constate que, depuis son départ, il n'a rien appris qui puisse « éclairer cette mystérieuse catastrophe " (Mérimée, 1927 : 38-39). Le narrateur dans Lokis recourt à un procédé biaiseur pour cautionner - en sourdine - la lecture indiciaire, confirmant la nature ursine du comte. Mais, du même coup, il fait diversion à la curiosité de son auditoire, en se lançant dans une explication savante destinée à " noyer le poisson " pour empêcher les autres personnages de poser les questions que se pose aussi le lecteur qui, de cette manière, reste sur sa faim (pour être confirmée, la nature anomale du comte n'en reste pas moins un mystère inexpliqué) :

- Mais, reprit-elle, pourquoi l'avez-vous intitulée Lokis ? Pas un seul des personnages ne s'appelle ainsi.

- Ce n'est pas un nom d'homme, dit le professeur. - Voyons, Théodore, comprenez-vous ce que veut dire Lokis?

- Pas le moins du monde.

- Si vous vous étiez bien pénétré de la loi de la transformation du sanscrit au lithuanien, vous auriez reconnu dans lokis le sanscrit arksha ou rikscha. On appelle lokis, en li-

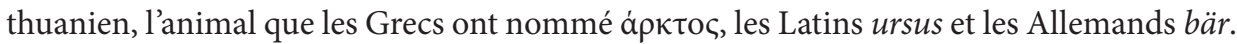

Vous comprenez maintenant mon épigraphe :

Miszka su Lokiu

Abu du tokiu.

Vous savez que, dans le roman de Renard, l'ours s'appelle damp Brun. Chez les Slaves, on le nomme Michel, Miszka en lithuanien, et ce surnom remplace presque toujours le nom générique, lokis. C’est ainsi que les Français ont oublié leur mot néolatin de goupil ou gorpil pour y substituer celui de renard. Je vous en citerai bien d'autres exemples.

Mais Adélaïde remarqua qu’il était tard, et on se sépara (Mérimée, 1993 : 262-263).

Dans cette optique, on peut analyser le récit fantastique comme un roman policier subverti. L'usage manipulateur des indices ${ }^{4}$ et des paralipses et l'effet de suspense

4. Dans son étude "Traces. Racines d'un paradigme indiciaire », Carlo Ginzburg définit le paradigme indiciaire comme un modèle épistémologique applicable, entre autres, au roman policier : «Dans les trois cas, des traces même infinitésimales permettent de saisir une réalité plus profonde, impossible à atteindre autrement. Des traces : plus précisément des symptômes (dans le cas de Freud), des indices (dans le cas de Sherlock Holmes), des signes picturaux (dans le cas de Morelli) (Ginzburg, 2010 : 232). 
narratif figurent parmi les topoï du roman policier, mais le fantastique les reprend, en les inversant. L'enquête policière apporte une solution rationaliste ${ }^{5}$ à l'énigme, tandis que le fantastique procède par irrationalisation. Le fantastique remplace la causalité infaillible du roman policier par une causalité incertaine, hypothétique. Aucune cohérence signifiante ne sera proposée au lecteur en dernière instance et si sa capacité déductive est sollicitée avec instance par le texte, aucune confirmation ne viendra sanctionner l'interprétation indiciaire à laquelle le lecteur se voit réduit. Dans cette optique, nous allons nous référer à l'analyse de Thomas Narcejac qui considère le fantastique comme « la tentation de tout auteur policier » $(1975: 121)$, dans la mesure où le fantastique " apparaît comme une explication bloquée, une explication qui ne peut aller jusqu'au bout parce qu'elle débouche sur l'impossible» (ibid. : 129). D'Hauteville, dans Fragoletta, tout comme le narrateur dans La Vénus d'Ille et celui de Lokis, s'improvisent enquêteurs, mais ils échouent, laissant en plan une enquête avortée. D'Hauteville parvient à résoudre certaines énigmes : l'identité de l'agresseur de Lillo, la nature des liens qui unissent Fragoletta et Savérelli, mais il ne réussit pas à percer le mystère de Camille et ne saura jamais que Camille et Philippe sont la même personne. Le narrateur, dans La Vénus d'Ille, opte pour un discours inquisitif minimaliste qui se disperse dans une description asyndétique de témoignages et de preuves qu'il n'ordonne pas. Multipliant les réticences et les ellipses, ce discours s'enfonce dans une objectivité faussée qui représente en fait une autocensure. Tantôt il essaye d'investir les indices d'un sens qui se révèle par la suite erroné (les traces sur le corps d'Alphonse), tantôt il les transforme en signifiants vides, en s'interdisant de les commenter (la bague retrouvée au pied du lit, les empreintes profondes dans le sol qui mènent à la statue). Voyant le sourire moqueur du procureur du roi, le narrateur interrompt l'interrogatoire du domestique :

Je demandai à cet homme si M. Alphonse avait sa bague de diamants lorsqu'il lui parla. Le domestique hésita pour répondre ; enfin, il dit qu'il ne le croyait pas, qu'il n'y avait fait du reste aucune attention.

- S'il avait eu cette bague au doigt, ajouta-t-il en se reprenant, je l'aurais sans doute remarquée, car je croyais qu'il l'avait donnée à Mme Alphonse.

En questionnant cet homme je ressentais un peu de la terreur superstitieuse que la déposition de Mme Alphonse avait répandue dans toute la maison. Le procureur du roi me regarda en souriant, et je me gardai bien d’insister (Mérimée, 1927 : 38).

Son enquête policière débouche ainsi sur le silence, sur un non-dit où se dessine

5. C'est-à-dire une explication univoque et exhaustive des mobiles et du modus operandi du criminel, qui intègre l'énigme à une intelligibilité infaillible du réel, faisant foi aux capacités intellectuelles du détective. Selon l'expression de T. Narcejac, le criminel dans les œuvres d'E. Poe, le créateur du genre, se présente comme « un automate logique » $(1975: 27)$, alors que le détective est « le lieu mental où la vérité se formule peu à peu » (ibid. : 30). 
en creux le sens manquant, refoulé en dehors du rationalisable et du concevable et, par conséquent, du dicible. Le même procédé se manifeste encore plus clairement dans Lokis :

Enfin, le valet de chambre apporta une barre de fer et nous enfonçâmes la porte... Non! Le courage me manque pour décrire le spectacle qui s'offrit à nos yeux. La jeune comtesse était étendue morte sur son lit, la figure horriblement lacérée, la gorge ouverte, inondée de sang. Le comte avait disparu, et personne depuis n’a eu de ses nouvelles.

Le docteur considéra l'horrible blessure de la jeune femme.

- Ce n'est pas une lame d'acier, s'écria-t-il, qui a fait cette plaie... C'est une morsure. (Mérimée, 1993 : 262)

Les points de suspension, signalant l'aposiopèse, et les points de conduite qui coupent court à l'enquête et au récit sont les marques d'une parole rationnelle qui se désagrège face à l'irrationalité absolue. Ce «laconisme mutilatoire», selon l'expression de Thierry Ozwald (1991 : 95), permet au narrateur d'agir comme un " constricteur de sens » (1991: 92), de produire, parallèlement au sens manifeste, un sens dissimulé, tu ou sous-entendu, qui sape la cohérence signifiante et qui constitue proprement un sens contraire, incompatible, un " contre-sens » (1991 : 93). Ce silence lourd de sens est caractéristique de l'écriture fantastique. Louis Vax affirme : "Nous entrons dans le roman policier pour avoir une explication et dans le conte fantastique pour n'en pas avoir» (1965: 102). Autrement dit, la dynamique de l'énigme dans le roman policier participe d'un sens qui se fait, alors que dans le fantastique l'explication boiteuse, entachée de non-dits, est génératrice d'un sens qui se défait.

En tout cas, cette analyse sans doute incomplète du rôle du silence dans les trois textes, aura permis de mettre en évidence un aspect essentiel de l'écriture fantastique en tant que projet esthétique. L'écriture fantastique se présente comme une manipulation discursive qui produit un univers fictionnel transgressif, un trompe-l'œil référentiel. Les trois œuvres qui se situent dans un cadre spatio-temporel réaliste et dans un contexte historique facilement identifiable, adoptent, en apparence, les conventions de l'illusion réaliste qui implique une adéquation entre le signe et l'objet fictionnel qu'il désigne. Les réticences explicatives sabordent cette illusion référentielle, car elles sont révélatrices d'une inadéquation entre le signe et l'objet qui, résistant à la réduction définitionnelle et explicative de l'inconnu au connu, oppose à la tentative de conceptualisation son opacité signifiante. Il serait peut-être utile de rappeler, à ce propos, qu'en latin le substantif silenda signifie, entre autres, «choses qu'on doit taire », « mystères», « secrets »

6. Gaffiot, Dictionnaire latin-français, article "Sileo ", http://www.lexilogos.com/latin/gaffiot. php?q=silen. 


\section{BIBLIOGRAPHIE}

Baroni R. 2007. La Tension narrative. Suspense, curiosité et surprise. Paris. Seuil.

Bozzetto R. 1998. Territoires des fantastiques. Aix-en-Provence. Université de Provence.

Corbin A. 2016. Histoire du silence de la Renaissance à nos jours. Paris. Albin Michel.

Crouzet M. 1984. Monstres et merveilles : poétique de l'Androgyne, à propos de Fragoletta. Romantisme. Vol. 14 No 45. 25-42 (www.persee.fr).

Fabre J. 1992. Le miroir de sorcière. Paris. José Corti.

Gaffiot. 1934. Dictionnaire français-latin. http://www.lexilogos.com/

Genette G. 1972. Figures III. Paris. Seuil.

Ginzburg C. 2010. « Traces. Racines d'un paradigme indiciaire». In Mythes, emblèmes, traces. Paris. Verdier. 218-294.

Latouche H. 1867. Fragoletta. Paris. Michel Lévy Frères http://gallica.bnf.fr ark:/12148/ bpt6k54705938.

Mérimée P. 1927. La Vénus d'Ille. In Carmen ; La Vénus d'Ille; Tamango. Paris. F. Rouff. 27-39. http://gallica.bnf.fr ark:/12148/bpt6k54966879.

Mérimée P. 1993. Lokis. In Carmen et autres nouvelles. Paris. Classiques Français Booking international. 205-263.

Narcejac T. 1975. Une machine à lire : Le Roman policier. Paris. Denoël/Gonthier.

Ozwald T. 1991. La nouvelle mériméenne : entre atticisme et mutisme. La Licorne 21. 91-102.

Picard M. 1954. Le Monde du silence. Paris. PUF (traduit de l'allemand par J.-J. Anstett).

Todorov T. 1970. Introduction à la littérature fantastique en France. Paris. Seuil.

Vax L. 1965. La séduction de l'étrange (étude sur la littérature fantastique). Paris. PUF.

\section{An eloquent silence: the narration and the fantastic in Latouche's Fragoletta, Mérimée's La Vénus d'llle and Lokis}

ABSTRACT: The paper analyses three works employing a similar narrative strategy which triggers tension between the explicable and inexplicable and an interpretative crisis that allows a fantastic reading of the fictional text. This strategy is related to the paradoxical status of the narrator, due to which the reader is faced with an incomplete elliptic account because of the former's reluctance (or impossibility) to offer a definite interpretation of the events.

Keywords: narrative strategy, interpretative crisis, fantastic reading, clue, paralipse. 